\title{
Recent results on charmed baryons at Belle
}

\author{
Nikolay Gabyshev ${ }^{* \dagger}$ \\ Budker Institute of Nuclear Physics \& Novosibisrk State University \\ E-mail: gabyshev@inp.nsk.su
}

We report measurement of the decays of the $\Omega_{c}^{0}$ charmed baryon into hadronic final states and observe excited $\Omega_{c}^{*}$ charmed baryons in the decay mode $\Xi_{c}^{+} K^{-}$including $\Omega_{c}(3000), \Omega_{c}(3050)$, $\Omega_{c}(3066)$ and $\Omega_{c}(3090)$. In addition, we report the first observation of the $\Xi_{c}(2930)^{0}$ charmedstrange baryon with a significance greater than $5 \sigma$ in its decay to $K^{-} \Lambda_{c}^{+}$in $B^{-} \rightarrow K^{-} \Lambda_{c}^{+} \bar{\Lambda}_{c}^{-}$decays. The branching fractions of the decays $\Lambda_{c}^{+} \rightarrow \Sigma^{+} \pi^{-} \pi^{+}, \Lambda_{c}^{+} \rightarrow \Sigma^{+} \pi^{+} \pi^{0}$ and $\Lambda_{c}^{+} \rightarrow \Sigma^{+} \pi^{0} \pi^{0}$ are also measured. The analyses are based on a data set recorded by the Belle detector from $e^{+} e^{-}$ collisions produced by the KEKB collider.

The 39th International Conference on High Energy Physics (ICHEP2018)

4-11 July, 2018

Seoul, Korea

*Speaker.

${ }^{\dagger}$ on behalf of Belle collaboration. 


\section{Introduction}

Belle detector [1] took about $1 \mathrm{fb}^{-1}$ mainly at $\Upsilon(4 S)$ energy for 10 years (October 1999 - June 2010 ) of operation time due to high luminosity (at that moment world record of $2.1 \times 10^{34} / \mathrm{cm}^{2} / \mathrm{sec}$ ) of KEKB $e^{+} e^{-}$collider ( $B$ factory) [2]. Although main target of Belle experiment was observation of $C P$ violation at $B$ mesons, contribution of Belle to study charm physics and physics of charmed baryons is significant.

Charmed baryons consist on heavy $c$ quark and two light $(u, d, s)$ quarks. Even $s$ quark is more than 10 times lighter than $c$ quark, what allow to develope, for example, heavy quark effective theory (HQET).

There are several mechanisms to produce charmed baryons at $B$ factories: inclusive production from continuum; exclusive production from continuum; $B$ meson decays via $b$ quark transition to $c$ quark; and also possible $B$ decays via $W$ boson with opposite flavor.

Some recent (by ICHEP2018) Belle results on charmed baryons are following.

\section{1. $\Lambda_{c}^{+} \rightarrow \Sigma \pi \pi[3]$}

The $\Lambda_{c}^{+}$is the lightest charmed baryon and plays a key role in the study of both strong and weak interactions. As $\mathscr{B}\left(\Lambda_{b}^{0} \rightarrow \Lambda_{c}^{+} \ldots\right) \sim 10 \%$, improved measurements of the $\Lambda_{c}^{+}$hadronic branching fractions help in study of heavy-flavor baryon production. Recent improved measurements of the $\mathscr{B}\left(\Lambda_{c}^{+} \rightarrow p K^{-} \pi^{+}\right)$by Belle and BESIII also push to improve the accuracy of related decays. The $\Lambda_{c}^{+} \rightarrow \Sigma \pi \pi$ decay is particularly interesting as it allows to extract $\Sigma-\pi$ scattering length and helps in study of $\Lambda(1405) \rightarrow \Sigma \pi$ resonance.

Belle measured branching fractions of $\Lambda_{c}^{+} \rightarrow \Sigma^{+} \pi^{+} \pi^{-}, \Sigma^{0} \pi^{+} \pi^{0}$ and $\Sigma^{+} \pi^{0} \pi^{0}$ decays with $\Sigma^{+} \rightarrow p \pi^{0}$ and $\Sigma^{0} \rightarrow \Lambda \gamma$ decays, and when $\pi^{0} \rightarrow \gamma \gamma$ and $\Lambda \rightarrow p \pi^{-}$. There was not analysis of the $\Lambda_{c}^{+} \rightarrow \Sigma^{-} \pi^{+} \pi^{+}$decay as $\Sigma^{-} \rightarrow n \pi^{-}$decay only. The $\Lambda_{c}^{+} \rightarrow p K^{-} \pi^{+}$decay was selected as relative reference and analized by the same way. The cut on $\Lambda_{c}^{+}$scaled momentum $p / p_{\max }$ reduces combinatorial background and follows that only inclusive $\Lambda_{c}^{+}$candidates from continuum remain. Further, Boosted Decision Tree (BDT) selector based on kinematical variables is used. The BDT selector is trained on MC with expected signal and background contributions.

To extract signal yields in a model independent way, Dalitz distribution is binned and independent fit of $\Lambda_{c}^{+}$invariant mass is performed in each bin. Binning for $\Lambda_{c}^{+} \rightarrow \Sigma^{0} \pi^{+} \pi^{0}$ decay, as example, and fit for the $\Lambda_{c}^{+}$in one bin are shown at Figure 1.
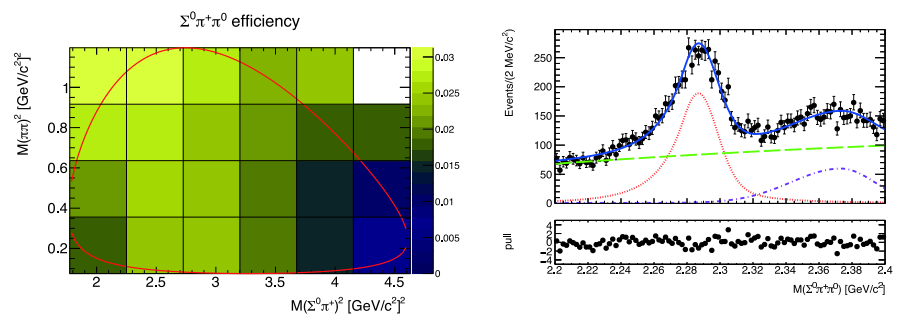

Figure 1: Dalitz distribution binning with reconstruction efficiency in bins and fit in one representative bin for the $\Lambda_{c}^{+} \rightarrow \Sigma^{+} \pi^{0} \pi^{0}$ decay.

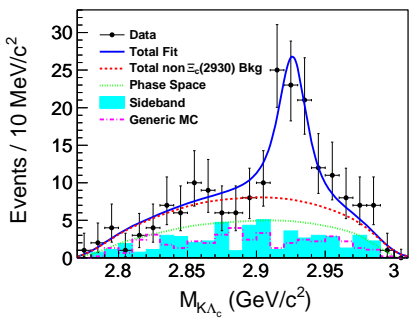

Figure 2: The $M_{K^{-} \Lambda_{c}^{+}}$distribution with $\Xi_{c}(2930)^{0}$ fit results for $B^{-} \rightarrow K^{-} \Lambda_{c}^{+} \bar{\Lambda}_{c}^{-}$decay. 
In results, branching fractions for $\Lambda_{c}^{+} \rightarrow \Sigma^{+} \pi^{-} \pi^{+}$and $\Sigma^{0} \pi^{+} \pi^{0}$ decays are improved by factor four. The $\Lambda_{c}^{+} \rightarrow \Sigma^{+} \pi^{0} \pi^{0}$ decay is measured for the first time.

\section{2. $B^{-} \rightarrow K^{-} \Lambda_{c}^{+} \bar{\Lambda}_{c}^{-}[4]$}

The $B^{-}$decay to $K^{-} \Lambda_{c}^{+} \bar{\Lambda}_{c}^{-}$was previously measured by Belle and BaBar with low statistics. And BaBar shows $\Xi_{c}(2930)^{0}$ state, but no significance. Also charmonium like state $Y(4630)$ was observed in $\Lambda_{c}^{+} \bar{\Lambda}_{c}^{-}$, which now is assumed to be the same state as $Y(4660)$ in $\pi^{+} \pi^{-} \psi^{\prime}$. The $Y(4660)$ is modeled to be $f_{0} \psi^{\prime}$ bound state. In that case it should have spin partner $Y_{\eta}$, which is $f_{0} \eta_{c}(2 S)$ bound state, with large decay width to $\Lambda_{c}^{+} \bar{\Lambda}_{c}^{-}$.

Current analysis is based on whole data taken at $\Upsilon(4 S)$ corresponding to 772 millions $B \bar{B}$ pairs. The $\Lambda_{c}^{+}$is reconstructed in $p K^{-} \pi^{+}, p K_{S}, \Lambda \pi^{+}, p K_{S} \pi^{+} \pi^{-}$and $\Lambda \pi^{+} \pi^{+} \pi^{-}$modes. The $B$ candidates are identified using beam-constrained mass, $M_{\mathrm{bc}}=\sqrt{E_{\mathrm{beam}}^{2}-\vec{p}^{2}}$, and mass difference, $\Delta M_{B}=M-m_{B}$, where $\vec{p}$ and $M$ are momentum in center-of-mass system (CMS) and invariant mass of $B$ candidate, $E_{\text {beam }}$ is the beam energy in CMS and $m_{B}$ is the nominal $B$ mass.

In the result observation of $\Xi_{c}(2930)^{0}$ with significance $>5 \sigma$ is reported. Figure 2 shows $M_{\Lambda_{c}^{+} \bar{\Lambda}_{c}^{-}}$distribution with $\Xi_{c}(2930)^{0}$ obtained mass and width. Branching fraction production of $\mathscr{B}\left(B^{-} \rightarrow \Xi_{c}(2930)^{0} \bar{\Lambda}_{c}^{-}\right) \times \mathscr{B}\left(\Xi_{c}(2930)^{0} \rightarrow K^{-} \Lambda_{c}^{+}\right)$is measured. No signal $Y(4660)$ nor its spin partner $Y_{\eta}(4613)$ is observed.

\section{Hadronic decays of $\Omega_{c}$ [5]}

$\Omega_{c}$ has less information than other charmed baryon, for example, no absolute branching fractions are measured, some decay modes are measured relatively normalizing mode $\Omega^{-} \pi^{+}$.

In this work we have looked for decays previously observed by CLEO and BaBar: $\Omega^{-} \pi^{+} \pi^{0}$, $\Omega^{-} \pi^{+} \pi^{+} \pi^{-}, \Xi^{-} K^{-} \pi^{+} \pi^{-}, \Xi^{0} K^{-} \pi^{+}$. The $\Sigma^{+} K^{-} K^{-} \pi^{+}$decay is previously observed in Fermilab with production more than $\Omega^{-} \pi^{+}$[6]. And also we looked for $\Xi^{-} K^{0} \pi^{+}, \Xi^{0} K^{0}, \Lambda K^{0} K^{0}$ decays not observed yet. The $\Omega^{-} \pi^{+}$decay is a reference. The $(\Xi / \Omega)^{-}$decay to $\Lambda(K / \pi)^{-}$respectively, the $\Xi^{0}$ decays to $\Lambda \pi^{0}$ and $\Sigma^{+}$to $p \pi^{0}$.

Figure 3 shows the invariant mass distributions for the eight decay modes under consideration. The masses in the fits are free parameters and the resultant masses are consistent with the world-average. Four of the modes presented here have been measured previously. In all cases, these new measurements are consistent with the previous measurements and provide substantial improvements in precision. For the cases where substructure is measured, the fraction of the primary mode is given. Three of the modes have been measured for the first time. It is surprising that we find a restrictive limit on the decay $\Omega_{c}^{+} \rightarrow \Sigma^{+} K^{-} K^{-} \pi^{+}$, which expected to be large. Also, whereas the other weakly decaying charmed baryons $Y_{c}$ have branching ratios $\mathscr{B}\left(Y_{c} \rightarrow Y \pi^{+} \pi^{-} \pi^{+}\right) / \mathscr{B}\left(Y_{c} \rightarrow Y \pi^{+}\right) \gg 1$, it is confirmed that, when $Y_{c}$ is an $\Omega_{c}^{+}$, this ratio is considerably less than 1 .

\section{Excited $\Omega_{c}^{*} \rightarrow \Xi_{c}^{+} K^{-}[7]$}

Until now, the $\Omega_{c}(2770) \rightarrow \Omega_{c} \gamma$ was the only excited state. Recently, LHCb discovered at $\Xi_{c}^{+} K^{-}$five narrow states $\Omega_{c}(3000 / 3050 / 3066 / 3090 / 3119)$ and wide enhancement at $3188 \mathrm{GeV}$ 

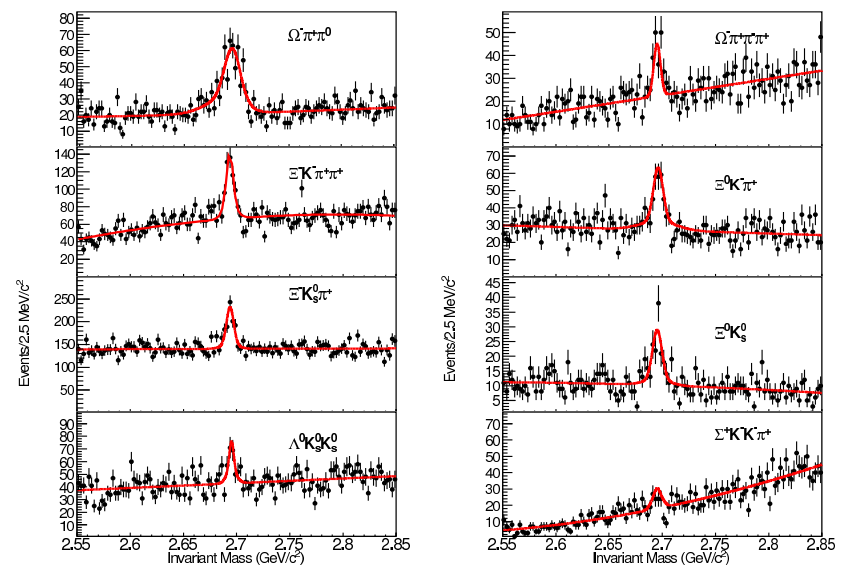

Figure 3: Invariant mass distributions for the eight $\Omega_{c}^{+}$decay modes.

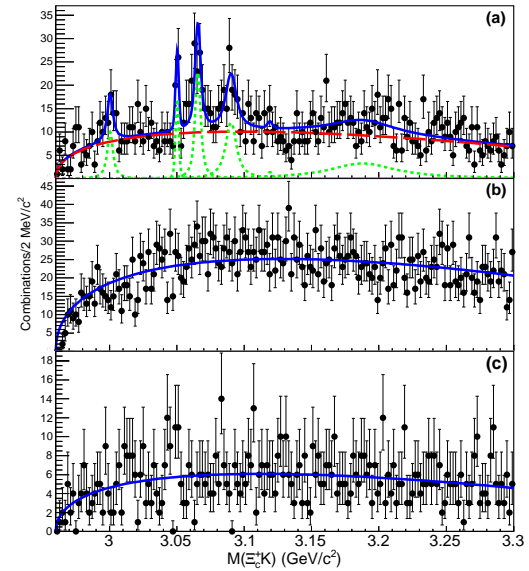

Figure 4: The $\Omega_{c}^{*} \rightarrow \Xi_{c}^{+} K^{-}$invariant mass distribution.

[8]. Belle have analized the same final states. The $\Xi_{c}^{+}$was reconstructed by decays of $\Xi^{-} \pi^{+} \pi^{+}$, $\Lambda K^{-} \pi^{+} \pi^{+}, \Xi^{0} \pi^{+}, \Xi^{0} \pi^{+} \pi^{-} \pi^{+}, \Sigma^{+} K^{-} \pi^{+}, \Lambda K_{S} \pi^{+}$and $\Sigma^{0} K_{S} \pi^{+}$.

The results are shown at Figure 4. Belle strongly confirmed $\Omega_{c}(3066)$ and $\Omega_{c}(3090)$ states, confirmed with less significance $\Omega_{c}(3000)$ and $\Omega_{c}(3050)$, but could not confirm $\Omega_{c}(3119)$, and has indicated enhancement at $3188 \mathrm{GeV}$.

\section{Summary}

There are still a lot of interesting to do with charmed baryons at Belle. And in the near future Belle will continue study of charmed baryons by its possibilities.

And in the not too far future, SuperKEKB [9] and Belle II [10] will make such studies with new opportunities due to large and good quality data. SuperKEKB is expected to have luminosity of 40 times of KEKB and will allow to Belle II to collect integrated luminosity by 2025 year of 50 times of Belle data.

\section{References}

[1] A. Abashian et al. (Belle Collaboration), Nucl. Instrum. Methods Phys. Res. Sect. A 479, 117 (2002).

[2] S. Kurokawa and E. Kikutani, Nucl. Instrum. Methods Phys. Res. Sect. A 499, 1 (2003).

[3] M. Berger et al. (Belle Collaboration), submitted to Phys. Rev. D, [hep-ex/1802 .03421]

[4] Y.B. Li et al. (Belle Collaboration), Eur. Phys. J. C 78, 252 (2018)

[5] J. Yelton et al. (Belle Collaboration), Phys. Rev. D 97, 032001 (2018).

[6] P. Frabetti et al. (E687 Collaboration), Phys. Lett. B 338, 106 (1994).

[7] J. Yelton et al. (Belle Collaboration), Phys. Rev. D 97, 051102(R) (2018).

[8] R. Aaij et al. (LHCb Collaboration), Phys. Rev. Lett. 118, 182001 (2017).

[9] Y. Ohnishi et al., Prog. Theor. Exp. Phys. 2013, 03 A011 (2013).

[10] T. Abe et al., [physics.ins-det/1011.0352]. 